\title{
BMJ Open Occupational radiation exposure and its health effects on interventional medical workers: study protocol for a prospective cohort study
}

\author{
Seulki Ko, ${ }^{1,2}$ Hwan Hoon Chung, ${ }^{3}$ Sung Bum Cho, ${ }^{4}$ Young Woo Jin, ${ }^{5}$ \\ Kwang Pyo Kim, ${ }^{6}$ Mina Ha, ${ }^{7}$ Ye Jin Bang, ${ }^{1,2}$ Yae Won Ha, ${ }^{1}$ Won Jin Lee ${ }^{1,2}$
}

To cite: Ko S, Chung HH, Cho SB, et al. Occupational radiation exposure and its health effects on interventional medical workers: study protocol for a prospective cohort study. BMJ Open 2017;7:e018333. doi:10.1136/ bmjopen-2017-018333

- Prepublication history for this paper is available online. To view these files, please visit the journal online (http://dx.doi. org/10.1136/bmjopen-2017018333).

Received 21 June 2017 Revised 30 September 2017 Accepted 30 October 2017

CrossMark

For numbered affiliations see end of article.

Correspondence to

Dr Won Jin Lee;

leewj@korea.ac.kr

\section{ABSTRACT}

Introduction Although fluoroscopically guided procedures involve a considerably high dose of radiation, few studies have investigated the effects of radiation on medical workers involved in interventional fluoroscopy procedures. Previous research remains in the early stages and has not reached a level comparable with other occupational studies thus far. Furthermore, the study of radiation workers provides an opportunity to estimate health risks at low doses and dose rates of ionising radiation. Therefore, the objectives of this study are (1) to initiate a prospective cohort study by conducting a baseline survey among medical radiation workers who involve interventional fluoroscopy procedures and (2) to assess the effect of occupational radiation exposure and on the overall health status through an in-depth cross-sectional study. Methods and analysis Intervention medical workers in Korea will be enrolled by using a self-administered questionnaire survey, and the survey data will be linked with radiation dosimetry data, National Health Insurance claims data, cancer registry and mortality data. After merging these data, the radiation organ dose, lifetime attributable risk due to cancer and the risk per unit dose will be estimated. For the cross-sectional study, approximately 100 intervention radiology department workers will be investigated for blood tests, clinical examinations such as ultrasonography (thyroid and carotid artery scan) and lens opacity, the validation of badge dose and biodosimetry.

Ethics and dissemination This study was reviewed and approved by the institutional review board of Korea University (KU-IRB-12-12-A-1). All participants will provide written informed consent prior to enrolment. The findings of the study will be disseminated through peer-reviewed scientific journals, conference presentations, and a report will be submitted to the relevant public health authorities in the Korea Centers for Disease Control and Prevention to help with the development of appropriate research and management policies.

\section{INTRODUCTION}

Medical radiation workers involved in interventional fluoroscopy procedures are exposed to higher radiation levels than those

\section{Strengths and limitations of this study}

- This study will provide comprehensive information on occupational radiation exposure and the health status of medical radiation workers involved in interventional fluoroscopy procedures.

- An in-depth cross-sectional study for interventional medical workers will provide a unique opportunity to investigate the overall health effects of radiation. A detailed questionnaire, laboratory and clinical examinations, badge monitoring and biodosimetry will be conducted to collect data.

- The major limitation of this study is the small number of participants for the in-depth crosssectional study.

who perform conventional radiography. ${ }^{1}$ However, this population is rarely studied as compared with other occupational fields or radiation epidemiology research. Therefore, epidemiological studies have been suggested, ${ }^{2}$ and an urgent need for implementing a culture of radiation protection has been called for regarding interventional fluoroscopy procedures. ${ }^{3}$ However, only a few studies have focused on investigating medical workers who perform or assist in interventional fluoroscopy procedures (table 1 ).

Previous studies on interventional medical workers have some limitations. ${ }^{4-16}$ No cohort study with active follow-up has been conducted on interventional medical workers except for the US Radiologic Technologists (USRT) cohort. $^{4-6}$ Only the Multispecialty Occupational Health Group (MOHG) study has attempted to investigate the long-term health effects of radiation on physicians performing interventional fluoroscopy procedures. ${ }^{78}$ Reported health outcomes have also focused on cataract development, whereas previous studies on the health effects of occupational radiation exposure primarily focused on 


\begin{tabular}{|c|c|c|c|c|}
\hline Study & Country & Enrolled population & Endpoint & Reference \\
\hline USRT study & USA & $\begin{array}{l}\text { Radiological technologists who } \\
\text { performed fluoroscopically } \\
\text { guided interventional procedures }\end{array}$ & $\begin{array}{l}\text { Mortality and incidence of cancer } \\
\text { and circulatory disease }\end{array}$ & $4-6$ \\
\hline MOHG study & USA & $\begin{array}{l}\text { Interventional cardiologists, } \\
\text { radiologists and } \\
\text { neuroradiologists }\end{array}$ & $\begin{array}{l}\text { Mortality from cancer and non- } \\
\text { cancer causes }\end{array}$ & 78 \\
\hline SCAI study & USA & $\begin{array}{l}\text { Interventional cardiologists and } \\
\text { staff }\end{array}$ & $\begin{array}{l}\text { Prevalence of orthopaedic injuries, } \\
\text { cataracts and cancer }\end{array}$ & 9 \\
\hline HCL study & Italy & $\begin{array}{l}\text { Interventional cardiologists and } \\
\text { staff }\end{array}$ & $\begin{array}{l}\text { Surrogate endpoints (chromosome } \\
\text { aberrations, telomere shortening, } \\
\text { CIMT and olfactory }{ }^{14} \text { dysfunction) }\end{array}$ & $10-12$ \\
\hline
\end{tabular}

CIMT, carotid intima-media thickness; EURALOC, European epidemiological study on Radiation-Induced Lens Opacities among interventional Cardiologists; HCL, Healthy Cath Lab; MOHG, Multispecialty Occupational Health Group; OCLOC, Occupational Cataracts and Lens Opacities in interventional Cardiology; RELID, Retrospective Evaluation of Lens Injuries and Dose; SCAI, Society for Cardiovascular Angiography and Interventions; SOLACI, Latin American Society of Interventional Cardiology; USRT, US Radiologic Technologists.

cancer and cardiovascular diseases. ${ }^{17} 18$ Only the Italian Healthy Cath Lab study used detailed biomarkers and relevant clinical approaches. ${ }^{10}$ Additionally, despite the variety of medical specialties involved in interventional radiological procedures, ${ }^{1}$ most studies have focused on the staff of interventional cardiology laboratories. Interventional cardiologists are probably the largest group and have the highest radiation exposure among interventional medical workers; however, a comprehensive approach is needed to understand the health effects of radiation exposure on the entire range of medical workers who are occupationally exposed owing to diverse interventional fluoroscopy procedures.

Therefore, additional well-organised epidemiological studies should be conducted to evaluate the precise risk of health outcomes, using measures expressed per unit of radiation dose. In particular, prospective cohort studies are necessary to determine the full extent of health risks among medical workers performing or assisting interventional fluoroscopy procedures. ${ }^{2}$ In addition, in-depth studies that include detailed questionnaire survey, clinical examinations and exploration of significant biomarkers would be helpful to have a better understanding of occupational radiation exposure and its health effects.

According to the extended usage of diagnostic radiation procedures, the number of medical radiation workers has been increasing in Korea. ${ }^{19}$ Interventional fluoroscopy procedures have also been widely used by several medical specialties, and the number of procedures performed is increasing ${ }^{2021}$; however, this high-risk group among medical radiation workers has not been monitored or investigated separately in Korea. We found a case report of radiation-induced necrosis in orthopaedic surgeon who performed interventional radiological procedures. ${ }^{22}$ We recently reported the work practices and the radiation exposure dose among male radiological technologists assisting with the fluoroscopy-guided interventional procedures. ${ }^{23}$ However, there was no research on the health effects of medical radiation workers who perform or assist the interventional fluoroscopy procedures in Korea.

Therefore, we have launched a study about the effects of radiation on medical workers involved in interventional fluoroscopy procedures. The objectives of this study are to present the study design and protocol of (1) cohort construction by enrolment of intervention medical workers with a baseline survey and (2) an in-depth cross-sectional study to identify occupational radiation exposure and overall health status.

\section{METHODS AND ANALYSIS}

\section{Study design and population}

The target population for this survey is all of the diagnostic medical radiation workers who perform or assist in interventional fluoroscopy procedures and are presently registered in the Korea Centers for Disease Control and Prevention (KCDC), which operates a lifetime management system of occupational radiation doses. The KCDC collects information of all diagnostic medical radiation workers including basic demographic data, workplace and radiation dose as part of a government-managed registry. The registry includes physicians (radiologists and other specialists), dentists, dental hygienists, radiological technologists, nurses and medical assistants. However, there is no direct information to distinguish the medical 
radiation workers who perform or assist in fluoroscopically guided procedures.

We will conduct two types of studies. First, as baseline, a cross-sectional study will be carried out with the support of various professional associations related to interventional radiological procedures. We will approach the study population through the professional societies and identify the target population based on the databases owned by each society. A cohort of interventional radiological workers will be set up with the voluntary participation of those who belong to the relevant professional societies, and they will be asked to complete the baseline questionnaire survey. We will compare between participants and the total membership of the societies regarding fluoroscopically guided procedures. After enrolment, we will combine the data from the questionnaires with dosimetry data supplied by the KCDC, which will also be linked to secondary health data, including the National Health Insurance (NHI) claim data, cancer registry and mortality data. The linked data will be annually updated to follow-up this cohort. We will estimate a lifetime attributable risk (LAR) of cancer for a given occupational exposure dose. Regarding long-term outcomes, standardised mortality ratios and incidence ratios will be calculated with secondary data linkage, and the dose-response relationship will be investigated by estimating excess relative risk (ERR) and excess absolute risk (EAR). Second, we will conduct an in-depth cross-sectional study with staff in the interventional radiology department. Because the first study yields crude exposure information only and requires a long follow-up period to detect the increased health risk, we build a small subcohort to detect potential clinical signs related to radiation health effects using an in-depth clinical study as a second study. This will include a detailed questionnaire survey, clinical examinations, a badge monitoring programme for validation of reported badge doses, biodosimetry and a review of past medical check-up records. The outline of the study design and data collection is presented in figure 1 .

\section{Baseline survey}

For the baseline survey, we work closely with the professional societies for workers who are involved in medical radiation intervention procedures (table 2). With endorsement from these professional societies, we will enrol medical radiation workers to set up a cohort. To enrol participants, we are conducting a self-administered questionnaire survey via visit in person or a web-based system (http://www.rhs.kr/intervention). The survey method will be different depending on the preference of each scientific society. With their cooperation, we will conduct an in-person survey at the periodic meetings for professional education and various conferences organised by the scientific societies. However, if only the web-based survey is allowed, we will promote the survey on the websites of the societies and via personal email. We will conduct a subsequent supplementary survey via telephone to obtain information regarding the questionnaire items that are left blank or answered insufficiently.

To maximise the participation rate, we will take several approaches, such as periodic contacts with the executives and publicity team of the relevant professional societies, asking them to link their website to the web survey, creating banner advertisements that promote the study on their websites, directly sending emails to introduce the web survey to individual members, reminder calls as follow-ups to invitations, raffle promotions to encourage participation in the in-person surveys and sending a statement from the KCDC for official cooperation to the related societies.

All intervention medical workers registered in the target societies will be contacted and invited to participate in the baseline survey. Previously, we conducted a survey of 15501 medical radiation workers in 2012$2013,{ }^{24}$ which represented about $26 \%$ of the total diagnostic medical radiation workers in Korea. Although this particular study mainly focused on radiological technologists, approximately $7 \%$ of diagnostic medical workers

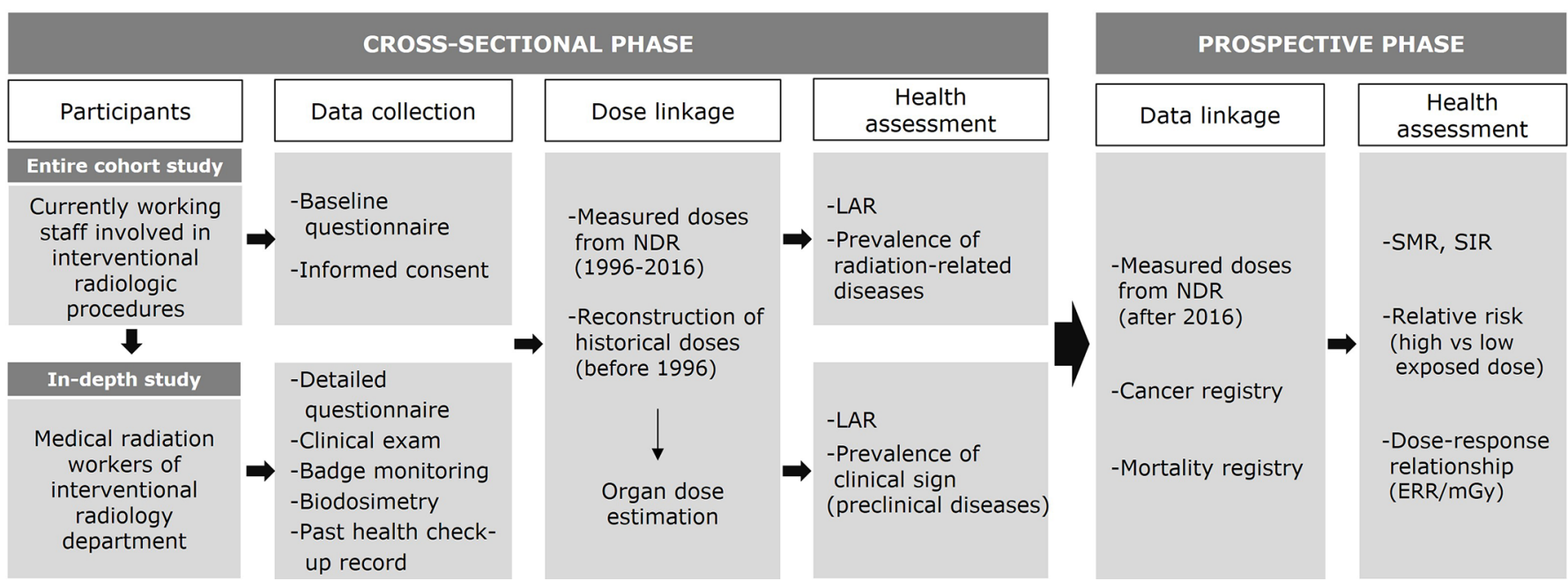

Figure 1 Study design and population. ERR, excess relative risk; LAR, lifetime attributable risk; NDR, National Dose Registry; SIR, standardised incidence ratio; SMR, standardised mortality ratio. 
Table 2 Target societies in Korea for the baseline survey

\begin{tabular}{|c|c|c|c|}
\hline Scientific societies & Member & Website & Specialty \\
\hline Korean Society of Interventional Radiology & Physicians & http://www.intervention.or.kr & - Interventional radiology \\
\hline Korean Society of Interventional Cardiology & Physicians & http://www.kvis.or.kr & Interventional cardiology \\
\hline $\begin{array}{l}\text { Korean Society of Interventional } \\
\text { Neuroradiology }\end{array}$ & Physicians & http://www.ksin.or.kr & $\begin{array}{l}\text { Interventional neurology } \\
\text { and neurosurgery }\end{array}$ \\
\hline Korean Pancreatobiliary Association & Physicians & http://www.kpba.kr & Gastroenterology \\
\hline Korean Orthopaedic Association & Physicians & http://www.koa.or.kr & Orthopaedic surgery \\
\hline Korean Minimally Invasive Spine Surgery Society & Physicians & http://komiss.org & Orthopaedic surgery \\
\hline Korean Pain Intervention Society & Physicians & http://www.korsis.or.kr & Pain and rehabilitation \\
\hline $\begin{array}{l}\text { Korean Society of Cardiovascular } \\
\text { Interventional Technology }\end{array}$ & Technologists & http://www.kscvit.or.kr & Interventional radiology \\
\hline $\begin{array}{l}\text { Korean Cardiovascular Technology } \\
\text { Association }\end{array}$ & Technologists & http://www.cta.or.kr & Interventional cardiology \\
\hline Korean Radiology Nurses Association & Nurses & - & Interventional procedures \\
\hline
\end{tabular}

reported that they had been involved in radiation interventional fluoroscopy procedures. Therefore, we assume that the number of interventional medical workers is approximately 4000 . This study is designed to possibly recruit all of the radiation medical intervention workers who are presently working; however, a sample size calculation is not appropriate at this time because there is no clear information to distinguish the medical radiation workers who perform or assist in fluoroscopically guided procedures and the main purpose of this study is to identify the possible target population at this stage.

\section{Questionnaire}

A questionnaire will be developed by reviewing previous cohort studies among radiation workers, adjusting the questionnaire items used for our previous survey of diagnostic radiological medical workers ${ }^{25}$ and conducting a pilot study among interventional medical radiation workers. The enrolment questionnaire includes items on demographics, work history, work practices, experience of high-dose exposure, radiation exposure by personal medical examination, health-related behaviours and medical history. The demographic data include the date of birth, gender, name and workplace address. Table 3 lists the information to be collected via the questionnaire survey. In addition, an informed consent form will be developed based on the Privacy Act in Korea; it will include items regarding the collection and use of personal information, identifying information and sensitive information, in addition to sharing of personal information with third parties and consent to participate in a research study.

Validation of self-reported medical radiation exposure and medical history

The medical radiation exposures, health-related behaviours and medical history included in the questionnaire will be validated through the NHI claim data. It is collected and managed by the National Health Insurance
Service (NHIS), the only public health insurance scheme in Korea, which covers the entire Korean population and includes eligibility data, the national health screening data and the healthcare usage data. ${ }^{26}$ We can use information on health-related behaviours from the national health screening data and information on medical radiation exposure and radiation-associated diseases from the healthcare usage data.

\begin{tabular}{|c|c|}
\hline $\begin{array}{l}\text { Domains (no of } \\
\text { questions) }\end{array}$ & Items \\
\hline Demographics (4) & $\begin{array}{l}\text { Date of birth, gender, name and } \\
\text { workplace address }\end{array}$ \\
\hline Work history (4) & $\begin{array}{l}\text { Job title, specialty, years since } \\
\text { beginning work and total duration of } \\
\text { work }\end{array}$ \\
\hline Work practices (7) & $\begin{array}{l}\text { Proportion of interventional } \\
\text { procedures for the recent year, } \\
\text { working days per month, working } \\
\text { hours per week, name of the main } \\
\text { procedure performed, badge } \\
\text { wearing, wearing of protective } \\
\text { equipment and use of shielding } \\
\text { devices }\end{array}$ \\
\hline $\begin{array}{l}\text { Experience of high } \\
\text { radiation exposure (2) }\end{array}$ & $\begin{array}{l}\text { Exposure to }>5 \text { milliSievert a quarter } \\
\text { and low WCC }\end{array}$ \\
\hline $\begin{array}{l}\text { Personal medical } \\
\text { examination (6) }\end{array}$ & $\begin{array}{l}\text { CT scan, fluoroscopy, nuclear } \\
\text { medicine imaging, PET-CT scan, } \\
\text { interventional radiography and } \\
\text { radiation therapy }\end{array}$ \\
\hline Lifestyle (2) & Smoking and alcohol consumption \\
\hline Medical history (9) & $\begin{array}{l}\text { Cataract, eye irritation, anaemia, } \\
\text { hypertension, dyslipidaemia, cancer, } \\
\text { thyroid disease, neck/back pain and } \\
\text { skin disease }\end{array}$ \\
\hline
\end{tabular}




\section{Data linkage and follow-up}

After the completion of the survey, participants' data will be linked with dosimetry data from the National Dose Registry managed by KCDC by means of participant's name, gender, date of birth and workplace address. The National Dose Registry contains the workers' name, gender, date of birth, personal identification number, workplace address, job title, quarterly measured dose data and the beginning and end of the period of measurement.

We will continue to evaluate the association between the radiation dose and its overall health effects with a longterm follow-up. Participants will be passively followed by linking the NHI claims data, Korea Central Cancer Registry (KCCR) and National Vital Statistics Registry that have been available since 2002, 1999 and 1991, respectively. We will use the healthcare usage data and the national health screening data in the NHI claim data to identify non-cancer diseases such as cataracts, cardiovascular diseases and thyroid diseases related to radiation exposure as well as other risk factors such as body mass index (BMI). The KCCR is the national level registry and maintains a high level of completeness (97.8\% in 2014). ${ }^{27}$ The registry data include cancer code (International Classification of Diseases and Related Health Problems, 10th Revision (ICD-10) and International Classification of Diseases for Oncology, Third Edition (ICD-O-3)), site, histological type, stage, diagnosis method and the date of diagnosis. The National Vital Statistics from Statistics Korea (http://kostat.go.kr) has also maintained a high level of completeness; the registration rate was $99.7 \%$ in $2014 .^{28}$ Mortality data are classified by the underlying cause of death according to the ICD-10.

To ascertain cancer incidence and the cause of death among study participants, personal identification numbers will be sent to the NHIS, Korean National Cancer Center and Statistics Korea; on our request, they will link these personal identification numbers to the NHI claims data, cancer registry data and mortality data. This linkage method is highly specific because of the uniqueness of the personal identification number of an individual in Korea, and we have successfully linked these data for radiological technologists previously. ${ }^{25}$ All the data linkage processes will be conducted only when informed consent is obtained.

\section{Calculation of radiation doses}

The KCDC has been carrying out monitoring programme for all medical radiation workers involved in diagnostic radiology since 1996. It maintains a centralised National Dose Registry and operates a lifelong follow-up management system for radiation dose in accordance with the Rules for Safety Management of Diagnostic Radiation and the Rules for Safety Management of Diagnostic Radiation Emitting Generators. ${ }^{29}$ Dose measurements have been collected quarterly by five personnel monitoring centres designated by the KCDC. The data for radiation dosimetry are available starting from 1996. To discover the occupational radiation exposure, individual doses recorded over the periods involved will be combined and annual effective doses and cumulative doses for each participant will be obtained as we reported previously. ${ }^{30}$

The organ dose estimation will be performed using the methodology applied in the USRT study. ${ }^{31}$ Briefly, the estimation of organ doses involves the use of measured badge dose and two ratios provided by the International Commission on Radiological Protection (ICRP): ${ }^{32}$ (1) the organ absorbed dose per unit of air kerma free-in-air (Gray per Gray) and (2) the personal dose equivalent per unit of air kerma free-in-air (Sievert per Gray). The calculation of organ absorbed dose in this study will use the ICRP factors and the organ dose coefficients. ${ }^{32}$ The equation is,

$$
\mathrm{D}_{\mathrm{T}}=\mathrm{H}_{\mathrm{p}}(\mathrm{d})\left[\frac{\mathrm{D}_{\mathrm{T}} / \mathrm{K}_{\mathrm{a}}}{\mathrm{H}_{\mathrm{p}}(\mathrm{d}) / \mathrm{K}_{\mathrm{a}}}\right]
$$

where $\mathrm{D}_{\mathrm{T}}$ is organ dose (Gray), $\mathrm{H}_{\mathrm{p}}(\mathrm{d})$ is badge dose (Sievert) and $K_{a}$ is air kerma free-in-air (Gray).

To adjust for the use of protective aprons and placement of the badge relative to the apron, we will apply the attenuation factor of protective device for apron. The radiation doses were not documented for individuals who were working before 1996; therefore, we will estimate their historical occupational exposed doses, by applying our previous methods, using a dose reconstruction model that includes predictors, such as age, gender and workplace. ${ }^{33}$

\section{Estimation of LAR of cancer}

The LAR of cancer specifies the probability that an individual will develop or die from cancer due to radiation exposure. ${ }^{34}$ For a given dose, LAR is the additional cumulated probability of having a specific cancer up to the maximum age of 89 years. We will calculate LAR based on the methods applied in the WHO report as follows. ${ }^{35}$ For an individual of sex $s$, exposed to dose $\mathrm{D}$ at age-at-exposure e and a specific cancer site at attained age $a$, the LAR is estimated as follows:

$$
\operatorname{LAR}(D, e, s)=\int_{e+L}^{a_{\max }} M(D, e, a, s) \frac{S_{a j}(a, s)}{S_{a j}(e, s)} d a
$$

To calculate LAR, a risk model is needed which can be either an ERR model or an EAR model, or a mixture of the two; $\mathrm{M}(\mathrm{D}, \mathrm{e}, \mathrm{a}, \mathrm{s})$ is the risk model in the equation. $\mathrm{S}_{\mathrm{aj}}$ $(\mathrm{a}, \mathrm{s})$ is the probability of cancer-free survival until age $a$ for the radiation-unexposed population; the ratio of $\mathrm{S}_{\mathrm{aj}}$ $(\mathrm{a}, \mathrm{s}) / \mathrm{S}_{\mathrm{aj}}(\mathrm{e}, \mathrm{s})$ is the conditional probability of an individual being alive and cancer-free at age-at-exposure $e$ to reach at least an attained age $a$. $\mathrm{L}$ is the minimum latency period depending on the cancer site. Survival functions $(\mathrm{S}(\mathrm{a}, \mathrm{s})$ or $\mathrm{S}(\mathrm{e}, \mathrm{s}))$ will be calculated based on the age-specific all-cause mortality rates derived from Statistics Korea for LAR of cancer mortality, while the adjusted survival functions $\left(\mathrm{S}_{\mathrm{aj}}(\mathrm{a}, \mathrm{s})\right.$ or $\left.\mathrm{S}_{\mathrm{aj}}(\mathrm{e}, \mathrm{s})\right)$ will be applied for LAR of cancer incidence, which are derived on the basis of all-cause mortality and the difference between all-cancer incidence and all-cancer mortality. ${ }^{35}$ 


\section{In-depth cross-sectional survey}

We will conduct a cross-sectional study for medical staff who work in the interventional radiology departments and will attend the 2017 Annual Joint Scientific Meeting of the Korean Society of Interventional Radiology, Korean Society of Cardiovascular Interventional Technology and Korean Radiology Nurses Association. These societies will provide detailed information, advertise and recruit volunteers who agree to participate by giving informed consent in advance. We aim to recruit approximately 100 workers, including 50 radiologists and 50 nurses and radiological technologists. The Korean Society of Interventional Radiology, the main collaborator of this project, is trying to recruit participants nationwide, through local branches of the society approaching whole list of 200 members with advance registration. The Korean Society of Cardiovascular Interventional Technology and Korean Radiology Nurses Association will select participants among the attendees of the Annual Joint Meeting.

The study contents are a detailed questionnaire-based survey, laboratory and clinical examinations, badge monitoring programme, biodosimetry and a review of the past health check-up records (table 4). A detailed questionnaire will give comprehensive information on the status of occupational radiation exposure and health status; clinical examinations and past heath check-up records could give us a clue about the health risks of radiation exposure regarding early warning signs. Using the badge monitoring programme and biodosimetry, we will investigate the validity of the reported badge dose and the correlation between physical dosimetry and biodosimetry.

\section{Detailed questionnaire}

A detailed questionnaire will be developed for the in-depth survey. We are reviewing previous cohort studies on radiation workers as a basis for developing the detailed questionnaire, and a pilot study will be conducted among the staff of the interventional radiology department of a hospital. While the baseline questionnaire we are developing inquires about the current radiation exposure, the detailed questionnaire consists of questions relating to work practices by calendar period in order to obtain comprehensive work-related information. The questionnaire includes information on demographics, work history, work practices, experience of high radiation exposure, management of radiation exposure, personal medical examination, health-related behaviours and medical history (table 4 ). The survey will be conducted during September of 2017 via the postal mail, together with a respondent-friendly description of the survey and the questionnaire. An informed consent form for the in-depth survey is prepared in the same way as that of the baseline survey.

\section{Clinical examination}

On-site clinical examinations, including anthropometry, blood pressure measurement, sampling for blood analysis and biodosimetry, ophthalmic examination and ultrasonography examinations, will be set up at the venue of the Annual Joint Scientific Meeting. All of the 100 registered participants will be contacted to schedule the examination during the meeting, and they will be provided with information regarding the aims, contents, methods and location of the temporal examination suite. All of the clinical examination procedures will be performed by trained medical personnel who will follow standardised protocols and use calibrated equipment. Based on the clinical and subclinical findings from these clinical examinations, potential radiation health risks could be detected, and this might increase our understanding of the initial damage from radiation exposure and allow us to infer long-term health outcomes. ${ }^{10}$

Anthropometrical profiles (ie, height, weight and waist circumference) will be measured as described by the Korea National Health and Nutrition Examination Survey (KNHANES), a health examination procedures manual. ${ }^{36}$ BMI will be calculated as the ratio of body weight (kilogram) to height squared (squared metre). Based on the standard protocol, systolic and diastolic blood pressures will be measured by trained nurses using a sphygmomanometer (JPN1 model; Omron, Kyoto, Japan) on the right arm of the seated subject after at least 5 min of rest.

Venous blood samples will be obtained from the antecubital vein by trained nurses to perform blood analysis and biodosimetry, and samples will be processed according to the KNHANES protocol. ${ }^{36}$ Blood will be drawn into several different tubes, such as an EDTA tube for testing complete blood count (CBC) and for analysing glycated haemoglobin; a serum separation tube for analysing blood lipid levels, high-sensitivity $\mathrm{C}$ reactive protein, homocysteine and thyroid function test; and a heparin tube for the biodosimetry sample. Detailed test items are listed in table 4 . Serum separating tubes will be kept at room temperature for $30 \mathrm{~min}$, and the blood will subsequently be centrifuged at 3000 revolutions per minute for $15 \mathrm{~min}$. EDTA tubes and heparin tubes will be mixed in a roller mixer for $10 \mathrm{~min}$. All blood samples will be refrigerated at $4^{\circ} \mathrm{C}$ and will be transported immediately to the accredited analytic laboratory (Seegene, Seoul, Korea).

Ophthalmic examinations will be conducted to investigate lens opacities, including a visual acuity test and a slit-lamp examination of the lens. A single ophthalmologist will conduct all of the examinations using the slitlamp BQ 900 (Haag-Streit AG, Koeniz, Switzerland). The diagnosis and grading of cataracts will be done according to the Lens Opacities Classification System (LOCS) III from early (stage 1 ) to severe (stage 5). ${ }^{37}$ The LOCS classification also describes the localisation of lens opacities (cortical, nuclear and posterior subcapsular).

Ultrasonography examination consisting of carotid artery and thyroid scans will be performed by a single radiologist, using a high-resolution B-mode ultrasonography (E-CUBE i7; Alpinion, Seoul, Korea) that has a linear 8-17 MHz transducer (L8-17; Alpinion) and the ability to save digital imaging and communications in medicine images to be retrospectively evaluated. Carotid 
Table 4 Items investigated with in-depth survey among the medical staff of intervention radiology department

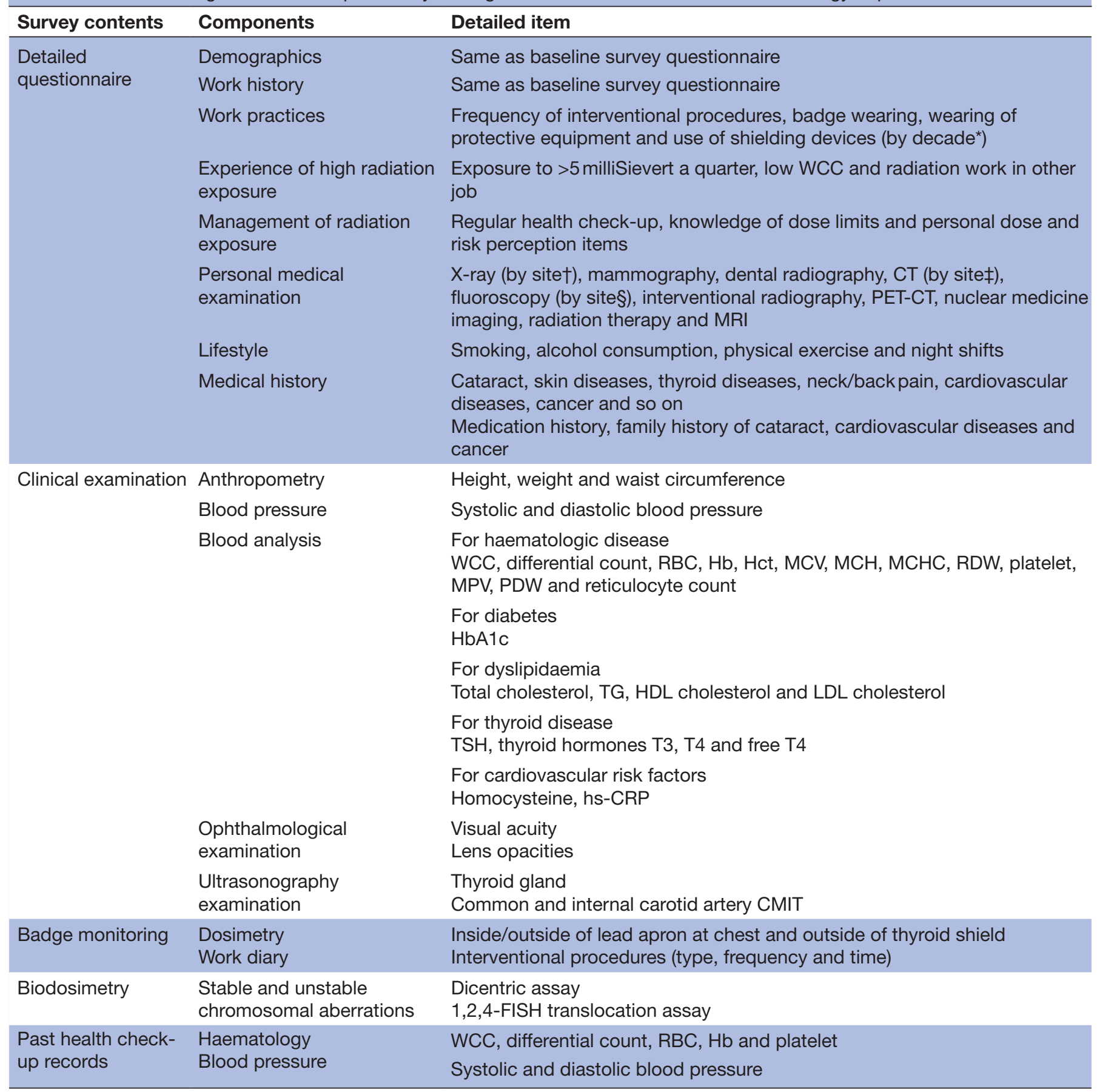

*1980-1989, 1990-1999, 2000-2009 and 2010-present. †Head and neck, chest, abdomen and extremity. †Head and neck, chest, abdomen, pelvis and extremity. $\S$ Stomach, intestine, hepatobiliary, kidney and others.

CIMT, carotid intima-media thickness; FISH, fluorescent in situ hybridisation; Hb, haemoglobin; HbA1c, glycated haemoglobin; Hct, haematocrit; HDL, high-density lipoprotein; hs-CRP, high-sensitivity C reactive protein; LDL, low-density lipoprotein; $\mathrm{MCH}$, mean corpuscular haemoglobin; MCHC, mean corpuscular haemoglobin concentration; MCV, mean corpuscular volume; MPV, mean platelet volume; PDW, platelet distribution width; PET, positron emission tomography; RBC, red blood cell; RDW, red cell distribution width; T3, tri-iodothyronine; T4, thyroxine; TG, triglyceride; TSH, thyroid-stimulating hormone; WCC, white cell count.

artery ultrasonography will be performed to measure carotid intima-media thickness (CIMT), which is a useful indicator for refining cardiovascular disease assessment among high-risk groups. ${ }^{38}$ The CIMT will be measured at near and far walls on both the left and right sides of the common carotid artery and internal carotid artery by automatic or semiautomatic measurement. Thyroid ultrasonography will detect thyroid nodules in both lobes, 
and the ultrasonography features of the nodules will be prospectively assessed in each participant during the examination. Subsequently, the nodules will be classified according to the Korean Thyroid Imaging Reporting and Data System ${ }^{39} 40$ to categorise thyroid nodules and stratify their malignancy risk.

\section{Validity of badge dose}

The validity of the badge doses reported from the National Dose Registry is important in evaluating occupational radiation exposure assessment and in estimating organ doses. To monitor the exact radiation exposure dose among interventional radiologists, study participants will wear three personal thermoluminescent dosimeters (Panasonic TLD system) inside and outside of the apron, and outside of the thyroid shield, always correctly for 1 month while keeping a working diary. Dose measurements will be collected by a fully accredited centre (Orbitech, Seoul, Korea). Measured badge doses will be compared with the reported dose data from the National Dose Registry in order to assess their validity. Intraclass correlation coefficients ${ }^{41}$ will be used as measures of validity.

\section{Biodosimetry}

Biodosimetry could be considered as an alternative method for estimating the absorbed dose, using a biomarker. Cytogenetic dosimetry analyses radiation-induced chromosome aberrations that are classified as unstable or stable aberrations, and this could evaluate individual radiation-related health risks represented by genomic instability. ${ }^{42}$ We will score dicentric chromosomes as unstable aberrations because this has been considered the most reliable method for biodosimetry. ${ }^{43}$ We will investigate reciprocal translocation as recommended in the case of prolonged exposure for a stable aberration. ${ }^{44}$ Blood samples for biodosimetry will be obtained during the clinical examination for the in-depth study. The samples will be collected in a heparin tube and will be processed for culturing within 24 hours after collection and delivery. The process of culturing, harvesting, staining and scoring for the analysis of dicentric chromosomes by solid Giemsa staining will be performed in accordance with the International Atomic Energy Agency recommendations. ${ }^{45}$ Metaphase cells will be prepared on a slide and 1, 2 and 4 whole chromosomes will be stained and scored for the analysis of translocation by fluorescence in situ hybridisation. The absorbed dose for each individual will be calculated from the measured yield of dicentrics and translocations, using dose-response calibration curves previously constructed at the Korea Institute of Radiological and Medical Sciences. ${ }^{46} 47$

\section{Past medical examinations}

Under the Korean law regarding the health protection of medical radiation workers (Medical Service Act, Article 37), registered medical radiation workers are required to wear personal thermoluminescent dosimeters and report their annual health check-up records, including the results of CBC test. We will ask participants to provide their previous health check-up data by filling out a structured form included in the in-depth questionnaire. The items include white cell count, red blood cells, haemoglobin, platelets, systolic and diastolic blood pressure and will be collected by requesting the electronic records of medical examination results. These data could be helpful in assessing the temporal trend of health effects from occupational radiation exposure.

\section{Statistical analyses}

All collected variables will be tabulated using summary statistics stratified by job title for continuous variables as mean values with SD and categorical variables as frequencies and percentages. The Student's t-test and the $\chi^{2}$ test will be used to test for the significance of the differences between two groups. The prevalence of clinical signs or diseases will be stratified by the job titles. Logistic regression analysis will be used to analyse binary variables for the abnormality of clinical examinations to ascertain whether occupational characteristics and radiation exposure are associated. Models will be adjusted for potential confounding factors, and the ORs and their 95\% CIs will be reported. Analysis of the long-term health effects through the follow-up will be conducted in parallel with the entire cohort.

\section{DISCUSSION}

This article has described the study design and protocol of a study on Korean medical radiation workers performing interventional fluoroscopy procedures. The advantage of this study compared with previous studies is that our study participants are linked to individual information by way of a questionnaire, radiation dosimetry, the NHI claims data, cancer registry and mortality data. All South Koreans are assigned a unique identification number at birth, and this number ensures accurate linkage to all national registry data. This allows investigating associations between radiation exposure and its health effects. The inclusion of an in-depth cross-sectional study examining a variety of preclinical health conditions is another unique advantage of this study. The study participants will provide detailed information on their work practices by calendar year. This allows for an in-depth exploration of occupational exposure and working conditions. We will collect participants' blood samples which enhance our ability to investigate radiation susceptibility and to assess exposure risk via surrogate biomarkers. Besides establishing scientific evidence of radiation-related health effects, this study will help to improve the awareness of the importance of radiation protection and to control the radiation exposure risk from interventional procedures. However, this study has the limitation of having a small number of participants for the in-depth cross-sectional study because of a limited budget. Further assessment by an international collaborative study would be necessary to 
overcome the limitation of small sample size. In addition, although the conditions of the ophthalmological examination may be less than perfect, examinations taking place at the venue of the Annual Joint Scientific meeting should be as quick and comfortable as possible. Therefore, to maximise the participant rate in this limited situation, we will set up the dark conditions enough to dilate the pupil during the ophthalmological examination without application of mydriatics.

Previous studies for intervention medical workers also had some limitations. The majority of studies were of a cross-sectional design and concentrated on cataract/ lens opacities; radiation risk was rarely assessed per unit of radiation dose. Compared with the previous studies, this study is rather unique because it collects comprehensive information to evaluate the health effects of low-dose radiation exposure. Therefore, this study will make important contributions to the literature by providing evidence regarding the occupational radiation exposure and its health effects on interventional medical radiological workers.

In summary, we will conduct a study regarding the health effects of radiation exposure on medical workers performing or assisting in interventional fluoroscopy procedures in Korea. This study features comprehensive information on the health outcomes, and the in-depth survey provides unique opportunities to investigate work-related factors and radiation exposure status of the interventional medical workers. This study will give further understanding of work practices and the association between protracted occupational radiation exposure and the health of interventional medical workers.

\section{Ethics and dissemination}

Informed written consent, including permission to collect personal information, and access to radiation dosimetry, NHI claims data, cancer registry and mortality data will be voluntarily obtained from each study participant before enrolment in the study. The participants of the baseline survey and in-depth study will receive a coupon for coffee (approximately worth US\$4) and a gift card (approximately worth US\$90), respectively.

The findings of the study will be shared with each professional society first and will be disseminated to their members through the society's website and its educational meetings. The main results of the study will also be disseminated through peer-reviewed scientific journals, and national and international academic conferences. We will also provide a full report to the KCDC, the organisation that is responsible for developing appropriate research and management policies.

\footnotetext{
Author affiliations

${ }^{1}$ Department of Preventive Medicine, Korea University College of Medicine, Seoul, South Korea

${ }^{2}$ Graduate School of Public Health, Korea University, Seoul, South Korea

${ }^{3}$ Department of Radiology, Korea University Ansan Hospital, Korea University College of Medicine, Ansan, South Korea
}

${ }^{4}$ Department of Radiology, Korea University Anam Hospital, Korea University College of Medicine, Seoul, South Korea

${ }^{5}$ National Radiation Emergency Medical Center, Korea Institute of Radiological and Medical Sciences, Seoul, South Korea

${ }^{6}$ Department of Nuclear Engineering, Kyung Hee University, Yongin, South Korea ${ }^{7}$ Department of Preventive Medicine, Dankook University College of Medicine, Cheonam, South Korea

Contributors SK and WJL: study concept and design, study coordination and drafting the manuscript. HHC and SBC: study design, planning of clinical examinations and revising the manuscript. YWJ: biodosimetry and revising the manuscript. KPK: badge monitoring and revising the manuscript. MH: study design, questionnaire and revising the manuscript. YJB and YWH: conducting field study, badge monitoring and revising the manuscript. All authors: approved and critically reviewed the final version of the manuscript.

Funding This work was supported by the Korea Ministry of Health and Welfare and Center for Disease Control and Prevention (grant no 2017E3600600) from 13 April 2017 to 12 February 2018.

Competing interests None declared.

Ethics approval Institutional Review Board of Korea University (KU-IRB-12-12-A-1).

Provenance and peer review Not commissioned; externally peer reviewed.

Open Access This is an Open Access article distributed in accordance with the Creative Commons Attribution Non Commercial (CC BY-NC 4.0) license, which permits others to distribute, remix, adapt, build upon this work non-commercially, and license their derivative works on different terms, provided the original work is properly cited and the use is non-commercial. See: http://creativecommons.org/ licenses/by-nc/4.0/

(C) Article author(s) (or their employer(s) unless otherwise stated in the text of the article) 2017. All rights reserved. No commercial use is permitted unless otherwise expressly granted.

\section{REFERENCES}

1. Valentin J. Avoidance of radiation injuries from medical interventional procedures. Ann ICRP 2000;30:7-67.

2. Linet MS, Kim KP, Miller DL, et al. Historical review of occupational exposures and cancer risks in medical radiation workers. Radiat Res 2010;174:793-808.

3. Bartal G, Roguin A, Paulo G. Call for implementing a radiation protection culture in fluoroscopically guided interventional procedures. AJR Am J Roentgenol 2016;206:1110-1.

4. Linet MS, Hauptmann M, Freedman DM, et al. Interventional radiography and mortality risks in U.S. radiologic technologists. Pediatr Radiol 2006;36 Suppl 2:113-20.

5. Rajaraman P, Doody MM, Yu CL, et al. Incidence and mortality risks for circulatory diseases in US radiologic technologists who worked with fluoroscopically guided interventional procedures, 1994-2008. Occup Environ Med 2016;73:21-7.

6. Rajaraman P, Doody MM, Yu CL, et al. Cancer risks in US radiologic technologists working with fluoroscopically guided interventional procedures, 1994-2008. AJR Am J Roentgenol 2016;206:1101-9.

7. Miller DL, Klein LW, Balter S, et al. Occupational health hazards in the interventional laboratory: progress report of the Multispecialty Occupational Health Group. J Vasc Interv Radiol 2010;21:1338-41.

8. Linet MS, Kitahara CM, Ntowe E, et al. Mortality in US physicians likely to perform fluoroscopy-guided interventional procedures compared with psychiatrists, 1979 to 2008. Radiology 2017;284:482-94.

9. Klein LW, Tra Y, Garratt KN, et al. Occupational health hazards of interventional cardiologists in the current decade: Results of the 2014 SCAI membership survey. Catheter Cardiovasc Interv 2015;86:913-24.

10. Picano E, Andreassi MG, Piccaluga E, et al. Occupational risks of chronic low dose radiation exposure in cardiac catheterisation laboratory: the Italian Healthy Cath Lab study. EMJ Int Cardiol 2013;1:50-8.

11. Andreassi MG, Piccaluga E, Gargani L, et al. Subclinical carotid atherosclerosis and early vascular aging from long-term low-dose ionizing radiation exposure: a genetic, telomere, and vascular ultrasound study in cardiac catheterization laboratory staff. JACC Cardiovasc Interv 2015;8:616-27. 
12. Andreassi MG, Piccaluga E, Guagliumi G, et al. Occupational health risks in cardiac catheterization laboratory workers. Circ Cardiovasc Interv 2016;9:e003273.

13. Jacob S, Boveda S, Bar O, et al. Interventional cardiologists and risk of radiation-induced cataract: results of a French multicenter observational study. Int J Cardiol 2013;167:1843-7.

14. Domienik J, Gryglak S, Jurewicz J. Characteristics of interventional cardiologists and their work practices for the study on radiationinduced lens opacities based on the methodology developed by ELDO-preliminary results. J Radiat Res 2016;57:431-7.

15. Ciraj-Bjelac O, Rehani M, Minamoto A, et al. Radiation-induced eye lens changes and risk for cataract in interventional cardiology. Cardiology 2012;123:168-71.

16. Vano E, Kleiman NJ, Duran A, et al. Radiation-associated lens opacities in catheterization personnel: results of a survey and direct assessments. J Vasc Interv Radiol 2013;24:197-204.

17. Richardson DB, Cardis E, Daniels RD, et al. Risk of cancer from occupational exposure to ionising radiation: retrospective cohort study of workers in France, the United Kingdom, and the United States (INWORKS). BMJ 2015;351:h5359.

18. Little MP. Radiation and circulatory disease. Mutat Res 2016;770:299-318.

19. Korea Centers for Disease Control and Prevention (KCDC). 2016. Occupational radiation exposure in diagnostic radiology in Korea: Centers for Disease Control and Prevention.

20. Jung SE, Kim KP, Dk H, et al. Research for National Medical Radiation exposure reduction infrastructure. Research for National Medical Radiation Exposure Reduction Infrastructure. KFDA/National Institute of Food and Drug Safety Evaluation. 2012.

21. Park H, Lim C, Kang B, et al. A study on the evaluation of patient dose in interventional radiology. J Radiol Sci Tech 2012;35:299-08.

22. Shim DM, Kim YM, Oh SK, et al. Radiation induced hand necrosis of an Orthopaedic surgeon who had treated a patient with fluoroscopyguided spine injection. J Korean Orthop Assoc 2014;49:250-4.

23. Yun JW, Cha ES, Ko S, et al. Work practices and radiation exposure among male radiologic technologists assisting fluoroscopically guided interventional procedures. Radiat Prot Dosimetry 2017:1-7. Epub ahead of print 3 Mar 2017.

24. Lee WJ, Ha M, Hwang SS, et al. Occupational radiation exposure and health effects in a cohort of diagnostic radiation workers in Korea. KFDA/National institute of food and drug safety evaluation. 2013.

25. Lee WJ, Ha M, Hwang SS, et al. The radiologic technologists' health study in South Korea: study design and baseline results. Int Arch Occup Environ Health 2015;88:759-68.

26. Cheol Seong S, Kim YY, Khang YH, et al. Data resource profile: the national health information database of the national health insurance service in South Korea. Int J Epidemiol 2017;46:799-800.

27. Jung KW, Won YJ, Oh CM, et al. Cancer statistics in Korea: incidence, mortality, survival, and prevalence in 2014. Cancer Res Treat 2017;49:292-305.

28. Statistics Korea. Cause of death statistics. http://kostat.go.kr/portal/ english/surveyOutlines/1/2/index.static (accessed 10 Jun 2017).

29. Korea Ministry of Health and Welfare. Rules for safety management of diagnostic radiation emitting generators. Health and welfare enforcement ordinance 3, 1995. Seoul. Korea: Ministry of Health and Welfare, 1995.

30. Lee WJ, Cha ES, Ha M, et al. Occupational radiation doses among diagnostic radiation workers in South Korea, 1996-2006. Radiat Prot Dosimetry 2009;136:50-5.
31. Simon SL, Weinstock RM, Doody MM, et al. Estimating historical radiation doses to a cohort of U.S. radiologic technologists. Radiat Res 2006;166:174-92.

32. International Commission on Radiological Protection (ICRP). Conversion coefficients for use in radiological protection against external radiation annals of the international commission on radiological protection. New York: Elsevier Science, 1996. Publication 74.

33. Choi Y, Kim J, Lee JJ, et al. Reconstruction of radiation dose received by diagnostic radiologic technologists in Korea. J Prev Med Public Health 2016;49:288-300.

34. National Research Council. Health risks from exposure to low levels of ionizing radiation: BEIR VII phase 2: National Academies Press, 2006.

35. World Health Organization (WHO). Health risk assessment: from the nuclear accident after the 2011 Great East Japan earthquake and tsunami: based on a preliminary dose estimation: World Health Organization, 2013.

36. Korea Centers for disease control and prevention (KCDC). Health examination procedures manual. Korea National Health and Nutrition Examination Survey(KNHANES). https://knhanes.cdc.go. $\mathrm{kr} / \mathrm{knhanes} / \mathrm{images} / \mathrm{board} / \mathrm{btn}$ download.pdf (accessed $10 \mathrm{Jun}$ 2017)

37. Chylack LT, Wolfe JK, Singer DM, et al. The lens opacities classification system III. The longitudinal study of cataract study group. Arch Ophthalmol 1993;111:831-6.

38. Stein $\mathrm{JH}$, Korcarz $\mathrm{CE}$, Hurst RT, et al. Use of carotid ultrasound to identify subclinical vascular disease and evaluate cardiovascular disease risk: a consensus statement from the American Society of Echocardiography Carotid Intima-Media Thickness Task Force. Endorsed by the Society for Vascular Medicine. J Am Soc Echocardiogr 2008;21:93-111.

39. Shin $\mathrm{JH}$, Baek JH, Chung J, et al. Ultrasonography diagnosis and imaging-based management of Thyroid Nodules: revised korean society of thyroid radiology consensus statement and recommendations. Korean J Radiol 2016;17:370-95.

40. Na DG, Baek JH, Sung JY, et al. Thyroid imaging reporting and data system risk stratification of Thyroid Nodules: categorization based on solidity and echogenicity. Thyroid 2016;26:562-72.

41. Shrout PE, Fleiss JL. Intraclass correlations: uses in assessing rater reliability. Psychol Bull 1979;86:420-8.

42. Pinto MM, Santos NF, Amaral A. Current status of biodosimetry based on standard cytogenetic methods. Radiat Environ Biophys 2010;49:567-81.

43. International Atomic Energy Agency. Cytogenetic analysis for radiation dose assessment 405 . Technical report. 2001.

44. Beinke C, Meineke V. High potential for methodical improvements of FISH-based translocation analysis for retrospective radiation biodosimetry. Health Phys 2012;103:127-32.

45. International Atomic Energy Agency. Cytogenetic dosimetry: applications in preparedness for and response to radiation emergencies. Vienna: IAEA-EPR, IAEA, 2011.

46. Cho MS, Lee JK, Bae KS, et al. Retrospective biodosimetry using translocation frequency in a stable cell of occupationally exposed to ionizing radiation. J Radiat Res 2015;56:709-16.

47. Jang S, Lee JK, Cho M, et al. Consecutive results of blood cell count and retrospective biodosimetry: useful tools of health protection regulation for radiation workers. Occup Environ Med 2016;73:694-700. 